\title{
Estilos de liderazgo y clima organizacional en la pequeña y mediana industria
}

\author{
César A. Valecillos *
}

\section{Resumen}

En este artículo, se presentan los resultados de un estudio exploratorio llevado a cabo el año de 1993 sobre el grado de asociación existente entre el liderazgo y el clima organizacional en los establecimientos industriales de la pequeña y mediana industria del Municipio Maracaibo del Estado Zulia. La indagación de campo se efectuó en una muestra de 74 establecimientos. La data se recolectó por medio de dos cuestionarios denominados: Estilo de Liderazgo y Clima Organizacional, diseñados por el autor. EI estudio arrojó como resultados la existencia de un grado de asociación estadísticamente significativo entre las variables analizadas, y en los estratos de ocupación (21-50) y (51-100), en que se clasificó la muestra seleccionada, se observó la tendencia de un estilo de liderazgo democrático y un clima organizacional amigable, mientras que en los estratos de ocupación (hasta 10) y $(11-20)$ un estilo de liderazgo de tendencia formal y un clima organizacional restrictivo.

Palabras claves:Estilo de liderazgo, Clima Organizacional, Pequeña y Mediana Industria, Venezuela.

\section{Leadership styles and organization environment at the small and medium enterprise}

\section{Abstract}

The aim of this paper is to present the results from an exploratory study carried out (in Maracaibo municipality) in order to prove the existing grade of association between

Reclbido: 30-10-96 Aceptado: 06-12-96

* Profesor Titular de la Facultad de Ciencias Económicas y Sociales de la Universidad del Zulia. Magister Scientiarum en Gerencia de Empresas (1992) Universidad Rafael Urdaneta. Maracaibo y Maestria en Desarrollo Organizacional (1994). Texas Austin University. USA y Universidad de Monterrey. Monterrey México. 
leadership and organization environment at the 74 industrial establishment of the small and medium enterprises of Maracaibo Municipality in 1993. The data was collected from two questionnnaires named: Leadership Styles and Organization Environment, designed by the author. The study found foun out the existence of a grade of association statiscally significant between the analyzed variables, and other stratus (21-50) and (51-100), out on leadership democratic of tendency and in the friendly organization environment, while in other stratums (until 10) and (11-20) a leadership style was revealed with formal tendency and restrictive organization environment.

Key words: Leadership Style, Organization Environment, Small and Medium Enterprise, Venezuela.

\section{Introducción}

A pocos pasos de la puerta que antecede el tercer milenio, América Latina y el Caribe enfrentan una encrucijada. Se trata, nada menos, de reencontrar el camino que garantice acceder al desarrollo pleno; un camino que parece haberse perdido debido a los acontecimientos del turbulento decenio que esta próximo a culminar. En el caso de nuestro país, la situación parece no ser la más halagadora si la comparamos con la de otros países que aparentemente dieran la impresión de haberlo encontrado y dado sus primeros pasos dentro del camino más adecuado e indicado. En lo que respecta al proceso de industrialización, las pequeñas y medianas industrias están llamadas a cumplir un papel de vanguardia y servir de base efectiva al resto del aparato productivo. A nivel macro, habrán de formularse políticas mas congruentes que posibiliten su consolidación de manera armónica; $y$, a nivel micro, fomentarse un liderazgo que siente las pautas hacia una cultura de desarrollo organizacional.

Se han efectuado diversos estudios orientados a realizar aportaciones a la gestión de los establecimientos industriales de la pequeña y mediana industria. En uno se observó la tendencia en el subsector de un liderazgo orientado a la tarea, sin considerar los efectos de los diferentes estilos en el ambiente laboral asf como repercusión en el desempeño de los trabajadores, por otra parte, el proceso administrativo y funcionamiento organizacional se desenvuelve con escaso criterio de previsión y planificación. (Apruzzeze, M. 1992; 9)

En la teoría de la organización y en las publicaciones especializadas sobre temas de gerencia, unos interesantes y otros restringidos, se observan diversas y encontradas definiciones acerca del concepto de liderazgo. Dentro de una perspectiva integradora, se le considera como una de las competencias fundamentales de todo administrador; reviste varias formas y constituye un proceso dentro de un continuo, a través del cual intenta influir sobre las actividades de un individuo o un grupo para lograr una meta en una situación dada, preocupándose tanto por las tareas como por las relaciones humanas. (BLANCHARD, Kent. 1993; 134). La misma situación priva so- 
bre el concepto de clima organizacional, configurándosele caracteristicas atinentes a la organización, a los individuos y la interacción entre ellos. Constituye el ambiente psicológico en el que se desenvuelven los trabajadores de la empresa, refiriéndose al ambiente de uno de sus subsistemas o la organización completa. (BATTEN, Joe. 1991; 13)

En un estudio experimental, considerado como uno de los más extensos acerca de la influencia del estilo de liderazgo sobre el clima organizacional, se encontró que cuando el líder hizo énfasis en los aspectos formales de la tarea, los individuos manifestaron percibir un ambiente de trabajo de sometimiento, conservador y frío emocionalmente. Cuando el líder orientó su comportamiento hacia la participación, los individuos manifestaron experimentar un ambiente reposado y amigable. Y cuando el líder dio prioridad al logro, la calidad y la productividad, los miembros manifestaron experimentar un ambiente de trabajo poco estructurado y elevado en lo que a recompensa de la iniciativa individual se refiere. (LITWIN y STRINGER, 1982; 73)

El propósito de la presente investigación, se centró entonces en explorar la existencia y magnitud del grado de asociación entre el liderazgo y el clima organizacional, dentro del ámbito de los establecimientos industriales de la pequeña y mediana industria, los cuales pueden ofrecernos indicios para el diseño de formas de intervención y planes estratégicos conducentes a la formación del nuevo rol que están llamados a desempeñar los socios propietarios y/o administradores, que sirvan a su vez de reforzadores $y$ guías de acción más eficientes para el personal a su cargo.

\section{El estudio y sus características generales}

Para dar respuesta al propósito del presente estudio se ejecutó una investigación con el siguiente objetivo general: Determinar la existencia de asociación entre los estilos de liderazgo de los socios propietarios y/o administradores, y el clima organizacional percibido por los trabajadores en los establecimientos industriales de la pequeña y mediana industria del Municipio Maracaibo del Estado Zulia, en Venezuela.

Este objetivo se satisfizo en la medida que se ejecutaron los siguientes objetivos especificos:

* Detectar los rasgos de los estilos de liderazgo del socio propietario $y / 0$ administrador y del clima organizacional percibido por los trabajadores, en los establecimientos industriales de la pequeña y mediana industria.

* Detectar si un estilo de liderazgo de tendencia formal, revela puntajes elevados en los indicadores de estructura y conflictos $y$, por el contrario, puntajes bajos en los indicadores de calidez, cooperación, progreso e iniciativa individual del clima organizacional.

* Detectar si un estilo de liderazgo de tendencia democrática, revela puntajes elevados en los indicadores de calidez y cooperación y, por ol contrario, puntajes bajos en los indicadores de estructura, conflictos, progreso $e$ iniciativa individual del clima organizacional. 
* Detectar si un estilo de liderazgo de tendencia hacia el apoyo, revela puntajes elevados en los indicadores progreso e iniciativa individual $y$, por el contrario, puntajes bajos en los indicadores de estructura, conflictos, calidez y cooperación del clima organizacional.

Detectar los rasgos de los estilos de liderazgo del socio propietario y/o administrador y del clima organizacional, percibido por los trabajadores en los establecimientos industriales que ocupan (hasta 10), entre $(11$ y 20) y $(21$ y 50$)$ trabajadores.

En este estudio, el estilo de liderazgo se consideró como la variable independiente y al clima organizacional como la variable dependiente. Se definió conceptualmente, el estilo de liderazgo, como: la competencia del que dirige en cuanto a sus actitudes, métodos, principios y valores, asi como en la habilidad para idear mejoras en la salud, el clima laboral, la innovación y la productividad de la organización, más que en la capacidad basada en las técnicas administrativas especfficas de planificación, programación y control. (SCOTT, Cyntia, 1993; 3) Y el clima organizacional como: el ambiente humano dentro del cual realizan sus oficios los trabajadores de una compañla, no se ve ni se toca, pero tiene una existencia real, al igual que el aire de una habitación rodea y afecta todo lo que sucede dentro de ella, es dinámico y varía a lo largo de un continuo pudiéndose percibir como favorable, neutro o desfavorable. (NEWSTRON, Jhon, 1990; 198)

Para hacer referencia a los rasgos de los estilos de liderazgo se conside- raron las dimensiones: formal, democrático y apoyo; y para caracterizar a cada uno de ellos los indicadores: supuestos administrativos, determinación de roles, promoción de la amistad, trabajo en equipo, valoración de la productividad y promoción de metas individuales. Mientras que para hacer referencia a los rasgos del clima organizacional se consideraron las dimensiones: restrictivo, amigable y enriquecedor, y para caracterizar a cada uno de ellos los indicadores: estructura, conflicto, calidez, cooperación, progreso e iniciativa individual.

Se definió operacionalmente el estilo de liderazgo como: el puntaje obtenido de los socios propietarios y/o administradores en el cuestionario diseñado por el autor, cuyos puntajes oscilaron entre 1 y 5 . Donde 1 y 2 revela una tendencia al estilo formal, 3 al estilo democrático y 4 y 5 al estilo de apoyo. En el caso de las dimensiones del estilo de liderazgo, se definieron operacionalmente como: estilo formal, puntaje obtenido en las respuestas con énfasis en los aspectos formales y normativos de la tarea, reflejándose en una puntuación entre 1 y 2 dentro del continuo. Estilo democrático, puntaje obtenido en las respuestas con énfasis al desarrollo de normas de amistad y relaciones interpersonales, reflejándose en una puntuación equivalente a 3 puntos dentro del continuo. $Y$ estilo de apoyo, puntaje obtenido en las respuestas con énfasis por la productividad e iniciativa individual, reflejándose en una puntuación entre 4 y 5 dentro del continuo.

El clima organizacional se definió operacionaimente como: el puntaje obtenido de los trabajadores en el cuestiona- 
rio diseñado por el autor, cuyos puntajes oscilaron entre 1 y 5 . Donde los dos primeros niveles revelan una tendencia a percibir un clima restrictivo, el nivel 3 un clima amigable y el nivel 4 y 5 un clima enriquecedor. En el caso de las dimensiones del clima organizacional, se definieron operacionalmente como: clima restrictivo, puntaje obtenido en las respuestas con connotaciones de rechazo al ambiente de trabajo, reflejándose en una puntuación entre 1 y 2 dentro del continuo. Clima amigable, puntaje obtenido en las respuestas con una predisposición humanizada hacia el ambiente de trabajo, reflejándose en una puntuación equivalente a 3 puntos dentro del continuo. $Y$ clima enriquecedor, colección de opiniones y creencias acompañado por una predisposición al crecimiento y desarrollo en el ambiente de trabajo, reflejándose en una puntuación entre 4 y 5 dentro del continuo.

\section{Metodología utilizada}

Dados los propósitos del estudio, se consideraron los siguientes aspectos metodológicos:

El nivel de análisis fue de tipo descriptivo, centrándose únicamente en conocer la asociación entre las variables, sin manipular el estado actual del fenómeno. El diseño utilizado fue de campo, procediéndose a recopilar los datos primarios directamente en la realidad mediante dos cuestionarios diseñados por el autor.

La población a la que se hizo referencia y sirvió de fuente para el muestreo estuvo conformada por el conjunto de 476 establecimientos de la industria manufacturera fabril (pequeña y mediana) existente en el Municipio Maracaibo del Estado Zulia y registrados en el Directorio Industrial Manufacturera Fabril de la Región Zuliana. (Conzuplan. O.C.E.I. 1989). Se hizo referencia a dicho directorio en vista de que facilitó el alcance pretendido en este estudio, ya que presenta a los establecimientos industriales ordenados según sus direcciones, ubicación geográfica y número de personas ocupadas. Distinguiendo a nivel de mediana industria un estrato superior que ocupa de (51 a 100) trabajadores y un estrato inferior que ocupa entre (21 y 50) trabajadores; de igual forma distingue a nivel de las pequeñas industrias un estrato superior que ocupa de (11 a 20) trabajadores y un estrato inferior que ocupa (hasta 10) trabajadores. Asimismo, considera la Clasificación Industrial Uniforme de Actividades Económicas Internacional. Código (C.I.I.U.) en atención a que presenta los establecimientos agrupados en diversas ramas, según su actividad económica, y en diferentes sectores industriales, destacándose: el tradicional, el intermedio, el metal mecánico y el grupo residual. Es de destacar que para determinar los establecimientos industriales que particularmente correspondían al Municipio Maracaibo se recurrió a la ayuda del departamento de cartografía del Consejo Zuliano de Planificación, dado que para el momento del inicio del presente estudio no existía registro oficial de la nueva división político territorial del Estado Zulia. De tal manera que se procedió a ubicar los establecimientos en la 19 parroquias del recientemente creado municipio Maracaibo a par- 
tir de sus direcciones domiciliarias, excluyéndose aquellos que caían fuera de los puntos de coordenadas: N1.192.000 E 209.400 HUSO 19 - N1.163.350 E 196.000 HUSO 19 y N1.192.000 E 196.000 HUSO 19 (CONZUPLAN. 1990; 112)

Elproceso de determinación muestral se basó en la técnica de muestreo aleatorio simple, el cual posibilitó que cada una de las unidades industriales tuviera igual probabilidad de ser escogida. La muestra seleccionada fue de 74 establecimientos, la misma se realizó por estratos de ocupación, así como por las diferentes ramas y sectores industriales en los que se clasifican los establecimientos industriales. La unidad piloto, la cual sirvió para probar la confiabilidad de los cuestionarios en su versión original, resultó ser de 14 establecimientos acatando la recomendación metodológica de seleccionar un $20 \%$ del número de establecimientos de la muestra.

Para proceder a recolectar los datos, el autor diseñó dos cuestionarios tipo escala Likert, denominados Estilo de Liderazgo y Clima Organizacional.

En cuanto al cuestionario Clima Organizacional, el mismo constituyó un instrumento de medición ordinal con un total de 60 reactivos o juicios en su versión original, elaborado con la finalidad de recoger la opinión de los trabajadores acerca de la percepción que tenian de sus ambientes de trabajo en las dimensiones del clima organizacional: restrictivo, amigable y enriquecedor, $y$ en los indicadores: estructura, conflicto, calidez, cooperación, progreso e iniciativa indivi-dual.
Para cada pregunta se presentaron cinco opciones de respuesta.

En el caso del cuestionario Estilo de Liderazgo, el mismo constituyó un instrumento de medición ordinal con un total de 62 reactivos o juicios en su versión original, elaborado con la tinalidad de recoger la opinión de los socios propietarios y/o administrador acerca de sus funciones y desempeño en las dimensiones del estilo de liderazgo: formal, democrático y apoyo, y en los indicadores: supuestos administrativos, determinación de roles, fomento de la amistad, relaciones interpersonales, valoración de la productividad y facilitar metas de crecimiento del estilo de liderazgo. Para cada pregunta se presentaron cinco opciones de respuesta.

Para probar la validez y confiabilidad de la versión original de ambos instrumentos se procedió a aplicarlos en los sujetos de los 14 establecimientos que conformó la prueba piloto. Las respuestas que dieron fueron sometidas a la técnicas de estadística inferencial:

Análisis de Correlación y de la Varianza para dos factores ANOVA. De dicho proceso resultaron eliminados 25 reactivos del cuestionario clima organizacional, quedando la versión final con un total de 35 preguntas, y del cuestionario estilo de liderazgo se eliminaron 20 ítemes, quedando la versión definitiva con un total de 42 juicios. Ambas versiones se aplicaron luego al resto de los sujetos de la muestra (70 establecimientos).

Para la tabulación de los datos, registrados en los cuestionarios, se construyeron dos tablas de doble entrada, una para cada variable. Dichas tablas se lle- 
naron con las puntuaciones dadas por los entrevistados, totalizándose las columnas para obtener los puntajes de los ítemes, y agrupándose por intervalos para hacer referencia a las dimensiones e indicadores de las variables. Con los totales de las columnas se obtuvieron los estadigrafos: media, varianza y desviación estándar correspondientes. Los productos de dichas tablas fueron la base del cálculo para el procesamiento estadístico y la obtención de los resultados.

\section{Influencia del liderazgo en el clima organizacional}

Mediante el método Análisis de Correlación, se procedió a analizar la relación existente entre las variables, así como sus correspondientes dimensiones e indicadores. En tal sentido, en la tabla No. 1 se presenta el producto del cruce de las dimensiones: formal, democrático y apoyo del estilo de liderazgo con los indicadores: estructura, conflicto, calidez, cooperación, progreso e iniciativa individual del clima organizacional;

observándose que la dimensión liderazgo formal reveló los puntajes más altos en los indicadores: estructura $(4,00)$ y conflicto $(3,00)$ y los más bajos en los de calidez $(1,00)$, cooperación $(1,50)$, progreso $(1,00)$ e iniciativa individual $(1,40)$ de la variable clima organizacional. Mientras que la dimensión liderazgo democrático reveló los puntajes más altos en los indicadores: calidez $(3,52)$ y cooperación $(4,52)$ y los más bajos en los indicadores: estructura $(1,00)$, conflicto $(1,10)$, progreso $(2,00)$ iniciativa individual $(2,60)$. Finalmente, la dimensión liderazgo de apoyo reveló los puntajes más altos en los indicadores: progreso $(4,84)$ e iniciativa individual $(5,00)$ y los más bajos en estructura $(1,35)$, conflicto $(2,53)$, calidez $(2,00)$ y cooperación $(2,20)$. Al observarse que los valores altos y bajos de la variable estilo de liderazgo hicieron pareja con los valores altos y bajos de la variable clima organizacional se determinó la existencia de una asociación lineal entre las mismas.

Para medir la magnitud o grado de asociación entre las variables se procedió a aplicar la prueba estadística Coeficiente de Correlación momento producto de Pearson. En la Tabla No. 2 se presentan los indices de correlación calculados para las dimensiones del estilo de liderazgo y los indicadores del clima organizacional.

Tabla No. 1

Correspondencia del clima organizacional con el estilo de liderazgo

\begin{tabular}{lccc}
\multicolumn{1}{c}{ Clima organizacional } & \multicolumn{3}{c}{ Estilo de liderazgo } \\
\cline { 3 - 4 } Indicadores & Formal & Democrático & Apoyo \\
\hline Estructura & $\mathbf{4 , 0 0}$ & 1,00 & 1,35 \\
Conflicto & $\mathbf{3 , 0 0}$ & 1,10 & 2,53 \\
Calidez & 1,00 & $\mathbf{3 , 5 2}$ & 2,00 \\
Cooperación & 1,50 & $\mathbf{4 , 5 2}$ & 2,20 \\
Progreso & 1,00 & 2,00 & $\mathbf{4 , 8 4}$ \\
Iniciativa individual & 1,40 & $\mathbf{2 , 6 0}$ & $\mathbf{5 , 0 0}$ \\
\hline
\end{tabular}


Tabla No. 2

Prueba estadistica de la correlación entre el clima organizacional y el estilo de liderazgo

\begin{tabular}{lccc}
\hline \multicolumn{1}{c}{ Clima organizacional } & \multicolumn{3}{c}{ Estilo de liderazgo } \\
\cline { 3 - 4 } Indicadores & Formal & Democrático & Apoyo \\
\hline Estructura & 0,96 & 0,96 & 0,94 \\
Conflicto & 0,94 & 0,96 & 0,94 \\
Calidez & & 0,93 & \\
Cooperación & & 0,95 & \\
Progreso & 0,96 & & 0,94 \\
Iniciativa individual & 0,91 & & 0,89 \\
\hline
\end{tabular}

Al oscilar los índices de correlación entre los valores 0,89 y 0,96 se determinó, además, que el grado de asociación entre ambas variables era significativo, razón por la cual se reveló la presencia de consistencia interna entre las mismas.

Para determinar que rasgos del estilo de liderazgo y del clima organizacional prevalecían en el ámbito de los establecimientos industriales de la pequeña y mediana industria se procedió a analizar los puntajes obtenidos en las respuestas re- gistradas en los cuestionarios. En la Tabla No. 3 se observa un valor total obtenido en la media de la variable estilo de liderazgo de $(3,12)$ y en la Tabla No. 4 un valor total obtenido en la media de la variable clima organizacional de $(2,93)$. Tomando en cuenta el rango de análisis (1-5) y los valores de las medias obtenidos, se evidenció la existencia de un estllo de liderazgo de tendencia democrático y un clima organizacional de tendencia amigable en la muestra seleccio-

Tabla No. 3

Estadigrafos de la variable estilo de liderazgo

\begin{tabular}{lccccc}
\hline \multicolumn{1}{c}{ Indicadores } & Items & Media & Varianza & Desv. Estandar \\
\hline Sup. Administ. & $1-7$ & & 2.55 & 2.38 & 1.54 \\
Determ. Roles & $8-17$ & 2.93 & 1.93 & 1.24 \\
Prom. Amistad & $18-24$ & 3.02 & 2.45 & 1.68 \\
Trab. Equipo & $25-30$ & 2.98 & 2.38 & 1.48 \\
Valor Product & $31-35$ & 3.62 & 1.62 & 1.30 \\
Pro. Met. Indiv. & $36-42$ & 3.65 & 2.91 & 1.70 \\
\hline Estilos & Items & Media & Varianza & Desv. Estandar \\
\hline Formal & $1-17$ & 2.74 & 1.99 & 1.41 \\
Democrático & $18-30$ & 3.00 & 2.54 & 1.59 \\
Apoyo & $31-42$ & 3.63 & 3.41 & 1.84 \\
\hline Variable & Items & Media & Varianza & Desv. Estandar \\
\hline Estilo Lideraz & $1-42$ & 3.12 & 2.36 & 1.62 \\
\hline
\end{tabular}


Tabla No. 4

Estadigrafos de la variable clima organizacional

\begin{tabular}{lcccc}
\multicolumn{1}{c}{ Indicadores } & Items & Media & Varianza & Desv. Estandar \\
\hline Estructura & $1-5$ & 2.41 & 1.84 & 1.36 \\
Conflicto & $6-11$ & 2.97 & 0.50 & 0.71 \\
Calidez & $12-16$ & 2.75 & 0.76 & 0.87 \\
Cooperación & $17-25$ & 3.12 & 1.88 & 1.37 \\
Progreso & $26-32$ & 2.50 & 3.30 & 1.81 \\
Prom. Individ. & $33-35$ & 3.81 & 0.26 & 0.51 \\
\hline Dimensiones & Items & Media & Varianza & Desv. Estandar \\
\hline Restrictivo & $1-11$ & 2.69 & 1.29 & 1.13 \\
Amigable & $12-25$ & 2.93 & 2.28 & 1.51 \\
Enriquecedor & $26-35$ & 3.15 & 3.24 & 1.80 \\
\hline Variable & Items & Media & Varianza & Desv. Estandar \\
\hline Clima Organiz & $1-35$ & 2.92 & 2.44 & 1.56 \\
\hline
\end{tabular}

nada. Por otra parte, mediante la técnica estadística Fischer, tales resultados se extrapolaron a la población con un $75 \%$ de correlación.

Finalmente, para detectar dentro de los estratos de ocupación en que se clasificó la muestra seleccionada los rasgos presentes en los estilos de liderazgo y clima organizacional se procedió a aplicar la técnica de estadística inferencial Análisis de la Varianza para dos factores ANOVA, con lo cual se midieron los efectos principales y de interacción de las variables dentro de los estratos de ocupación. En la Tabla No. 5 se presentan los resultados de dicho proceso, donde al determinarse para los efectos principales de la variable estilo de liderazgo una $F$ (Fisher) calculada de 0,13 se evidenció la existencia de diferencias significativas de los estilos de liderazgo dentro de los estratos de ocupación; prevaleciendo en los estratos de ocupación (hasta 10) y (1120) el estilo de tendencia al liderazgo formal y en los estratos de ocupación (21-50) y en el estrato de ocupación (51100) el estilo de liderazgo democrático. Por su parte, en cuanto al clima organizacional al determinarse una $\mathrm{F}$ calculada de 5,16 se detectó la existencia de diferencias significativas de los climas organizacionales dentro de los estratos de ocupación; prevaleciendo un clima restrictivo en los estratos de ocupación (hasta 10) y $(11-20)$ y un clima amigable en los estratos de ocupación (21-50) y (51-100). En cuanto a los efectos de interacción, al obtenerse se una F calculada de 40,062 se comprobó la existencia de interacción entre los estilos de liderazgo y el clima organizacional dentro de los estratos de ocupación.

\section{Conclusiones}

En el presente estudio se logro determinar la existencia de un grado de asociación significativo entre el estilo de 
Tabla No. 5

Resultado del análisis de la varianza ANOVA entre los estratos de ocupación

Fuente de variación
(Df)

Grados de

libertad
(Ms)

Variabilidad

entre los grupos estadísticamente

(Fc) $\mathrm{F}$

Calculada

Efectos principales

Estilo de liderazgo

Clima organizacional

Efectos de interacción
1

3

3
0,14

33,20
0,13

5,16

(EL) $\times(\mathrm{CO})$

40,06

\section{Dentro de los grupos}

liderazgo y el clima organizacional, razón por lo cual se infiere que los socios propietarios y/o administradores de los establecimientos industriales de la pequeña y mediana industria del Municipio Maracaibo del Estado Zulia influyen con sus acciones en el ambiente de trabajo percibido por los trabajadores.

En cuanto a los rasgos presentes en el estilo de liderazgo y clima organizacional de los establecimientos industriales de la pequeña y mediana industria del Municipio Maracaibo del Estado Zulia se evidenció que predomina un estilo de liderazgo de tendencia democrático y un clima organizacional de tendencia amigable.

De acuerdo a los resultados obtenidos se puso de manifiesto que el estilo de liderazgo de tendencia formal reveló puntajes altos en los indicadores de estructura y conflicto y bajos en los de calidez, cooperación, progreso e iniciativa individual del clima organizacional. El estilo de liderazgo de tendencia demo-
$27 \quad 1,08$

crático reveló puntajes altos en los indicadores calldez y cooperación y bajos en los de estructura, conflicto, progreso e iniciativa individual del clima organizacional. Finalmente, el estilo de liderazgo de tendencia hacia el apoyo arrojó puntajes altos en los indicadores progreso e iniciativa individual y bajos en los de estructura, conflicto, calidez y cooperación del clima organizacional. Con cual es válido advertir que fueron coincidentes con los hallazgos de los estudios de Litwin y Striger en 1968.

Con arreglo a los resultados se evidenció que en los estratos de ocupación (21-50) y (51-100) prevalece un estilo de liderazgo de tendencia democrático y un clima organizacional de tendencia amigable, mientras que en los estratos de ocupación (hasta 10) y (1120) prevalece un estilo de liderazgo de tendencia formal y un clima

organizacional de tendencia restrictivo. Se infiere que por el número de personas ocupadas y la visibilidad de la 
autoridad el liderazgo ejercido es de índole personal y afectivo influyendo en un ambiente de trabajo o clima donde las relaciones directas fungen de elemento cohesionador hacia los requerimientos de la organización.

\section{Consideraciones finales}

A pesar de lo exhaustivo del procesamiento estadistico y la consistencia metodológica que privo en el presente estudio, los resultados deben ser interpretados como una forma descriptiva de comparar la correlación obtenida con el valor que esperaríamos bajo circunstancias preponderantes y no como una relación fuerte entre las mismas, sobre todo si se toma en cuenta que se presentaron algunos inconvenientes durante el trabajo de campo. Por otra parte, dada la naturaleza de las variables analizadas se debería considerar una metodología de enfoque cualitativo que aborde la indagación desde una perspectiva sistémica y gestáltica, con una actitud de mente abierta y sin ideas preconcebidas, de modo de aprender a partir de la interacción con el objeto estudiado; los resultados podrían ser más ricos y adecuados a la realidad compleja. De ahí la necesidad de llevar a cabo otras pruebas que contrasten y reafirmen los resultados obtenidos y el empleo de otros enfoques.

Los socios propietarios y/o administradores de los establecimientos industriales de la pequeña y mediana industria deben tomar conciencia de la influencia que pueden ejercer en su personal a partir de las pistas y pautas que reflejan en los ambientes de trabajo, los cuales pueden repercutir favorablemente o no en su desempeño. La motivación al logro y calidad en el desempeño es un proceso complejo y multifactorial. No depende solo de factores externos, incluyendo el clima organizacional; fundamentalmente son intrínsecos a los individuos. Sin embargo dentro del marco de condiciones a planificar para el tan anhelado comportamiento productivo de los individuos, el administrador a todos los niveles podría constituirse en agente de cambio y facilitador del aprendizaje mediante un estilo de liderazgo de tendencia hacia el apoyo que fomente un clima organizacional enriquecedor.

En tal sentido, se hace necesario para el subsector de la pequeña y mediana industria instrumentar intervenciones planificadas para su desarrollo organizacional que ponga en el centro del proceso al ser humano y sus necesidades vitales. Se requiere del diseño de programas de formación y capacitación en aprendizaje organizacional donde la materia prima tiene que ver con aspectos relacionados con el liderazgo transformacional, el clima organizacional, la visión sistémica, y la formación de equipos, entre otros. En la fase inicial se ha de orientar, sensibilizar y convenir con los empresarios la necesidad de invertir recursos, energia y compromiso en el aprendizaje de estos aspectos, los cuales son vías más expeditas para una adecuación proactiva a las condiciones presentes y futuras que demanda la modernización de nuestra economía, los retos de la globalización y la inserción en el nuevo orden mundial. 


\section{Referencias Bibliográficas}

Apruzzece, María. (1992). La pequeña y mediana industria como generadora de empleo. La Universidad del Zulia. 9 págs.

Batten, Joe. (1991). Cómo construir una cultura de Calldad Total. Grupo Editorial lberoamericana. México. 13 págs.

Blanchard, Kent. (1993). Eatilo eficaz de dirlgir. Liderazgo Situacional. I.D.H. Ediciones. 134 págs.

CONZUPLAN. (1989). Directorio de la Industrla Fabril Pequeña y Mediana en la Región Zuliana. Maracaibo. Venezuela. Notas.

CONZUPLAN. (1990). Maracalbo. División Político Territorial. 112 págs.

Newstron, Jhon, (1990) Comportamiento Organizacional. Edit. Mc. Craw Hill. México. 198 págs.
Litwin y Stringer. (1982). Motivation and Organizational Climate. Boston: División of Reserch Harvard Busines Scholls. 73 págs.

Scott, Cyntia (1993). Cómo dirigir el camblo en las organizaclones. Grupo Editorial Iberoamericana, SA de CV. México. 3 págs.

Valecillos, César. 1991. Estilos de liderazgo y clima organizacional en la Pequeña y Mediana Industria. Tesis de Maestría. Universidad Rafael Urdaneta. 65 págs.

Valecillos, César, 1988. Cómo persuadir a un empresario para implementar la Administraclón por Objetivos y Resultados. Trabajo de ascenso Prof. Asociado Universidad del Zulia. 32 págs. 